Objectives: In RA patients in longstanding clinical and radiographic remission, achieved by a DAS28-driven T2T strategy, to investigate if US signs of inflammation differs between RA patients, treated with csDMARD or bDMARD (+/- csDMARD). Methods: Eighty-seven patients with RA in longstanding clinical (continuous DAS28 $<2.6$ for the preceding year) and radiographic (no progression for at least 1 year) remission, were included in the study. US of elbows, wrists, MCP2-5, knees, ankles and MTP2-5 were performed using a GE LOGIQE9 US unit. Each joint was scored for grey-scale synovitis (GSS) and synovial color Doppler activity (CDA) by a $0-3$ semi-quantitative score. Ultrasound remission was defined in two ways: either no $(G S S=0$ and $C D A=0$ ) or minimal $(G S S \leq 1$ and $C D A=0$ ) inflammation in any of the 24 assessed joints.

Results: Clinical characteristics and US findings are shown in the table. All 87 patients fulfilled DAS28 remission criteria at entry and CDAI remission was fulfilled in $76 \%$ and $79 \%$ in the csDMARD and bDMARD group, respectively. Complete absence of any signs of US inflammation (GSS $=0$ and $C D A=0$ ) was seen in $0 \%$ and $14 \%$ in the csDMARD and bDMARD groups, respectively $(p=0.01)$, while minimal US inflammation (GSS $\leq 1$ and $C D A=0$ ) was seen in $33 \%$ and $40 \%$ (NS). CDA in at least one joint was seen in the majority of patients in both groups, $58 \%$ and $57 \%$ respectively.

Table 1

\begin{tabular}{lccc}
\hline & csDMARD $(\mathrm{n}=45)$ & bDMARD $(\mathrm{n}=42)$ & \\
\hline Females & $28(62 \%)$ & $28(67 \%)$ & $\mathrm{NS}$ \\
Age (years) & $64(31-82)$ & $57(25-82)$ & $\mathrm{NS}$ \\
Disease duration & $6(1-44)$ & $12(0-54)$ & $\mathrm{p}<0.01$ \\
lgM-RF/anti-CCP positive & $25(56 \%) / 25(56 \%)$ & $28(67 \%) / 33(79 \%)$ & $\mathrm{NS} / \mathrm{p}<0.05$ \\
Erosive disease & $23(51 \%)$ & $34(81 \%)$ & $<0.01$ \\
Tender joint count & $0(0-1)$ & $0(0-1)$ & $\mathrm{NS}$ \\
Swollen joint count & $0(0-2)$ & $0(0-2)$ & $\mathrm{NS}$ \\
C-reactive protein (mg/L) & $4(1-13)$ & $5(4-26)$ & $\mathrm{p}<0.01$ \\
DAS28 & $1.7(1.1-2.4)$ & $2.0(1.6-2.5)$ & $\mathrm{p}<0.01$ \\
CDAl & $1.4(0-5.3)$ & $1.8(0-7.7)$ & $\mathrm{NS}$ \\
GSS-score (0-72) & $4(1-18)$ & $6(0-18)$ & $\mathrm{NS}$ \\
CDA-score (0-72) & $0(0-12)$ & $0(0-7)$ & $\mathrm{NS}$ \\
US minimal inflammation & & & \\
(GSS $\leq 1$ \& CDA=0) & $15(33 \%)$ & $17(40 \%)$ & $\mathrm{NS}$ \\
US no inflammation & & & \\
$\quad$ GSS $=0$ \& CDA=0) & $0(0 \%)$ & $6(14 \%)$ & $\mathrm{p}=0.01$ \\
\hline
\end{tabular}

Values are given as numbers (percentages) and median (range). Fisher's exact or Mann-Whitney test used for comparisons.

Conclusions: The majority of RA patients, in this cohort of patients in longstanding clinical and radiographic remission obtained through a DAS28 driven T2T strategy, had signs of inflammation as assessed by US, irrespective of receiving biologic treatment or not. For patients in clinical remission, the consequences of sustained US inflammation still have to be investigated.

References:

[1] Brown AK et al.: Arthritis Rheum 2006;54(12):3761-73.

[2] Saleem B et al: : Arthritis Rheum 2009;60(7):1915-22.

[3] Gandjbakhch F et al.: J Rheumatol 2011;38(9):2039-44.

Disclosure of Interest: None declared

DOI: 10.1136/annrheumdis-2017-eular.3763

\section{FRI0629 MAGNETIC RESONANCE IMAGING (MRI) INFLAMMATION OF THE FEET DEMONSTRATES SUBCLINICAL INFLAMMATORY DISEASE IN CUTANEOUS PSORIASIS PATIENTS WITHOUT CLINICAL ARTHRITIS}

A.J. Mathew ${ }^{1}$, P. Bird ${ }^{2}$, A. Gupta ${ }^{3}$, R.E. George ${ }^{3}$, D. Danda ${ }^{1} .{ }^{1}$ Clinical Immunology \& Rheumatology, Christian Medical College, Vellore, India; ${ }^{2}$ University of New South Wales, Sydney, Australia; ${ }^{3}$ Dermatology, Christian Medical College, Vellore, India

Background: Up to $40 \%$ of patients with cutaneous psoriasis may develop psoriatic arthritis (PsA). Early detection of PsA by advanced imaging techniques results in better response to therapy. There are very few studies evaluating the MRI appearance in feet of patients with psoriasis and PsA.

Objectives: This study sought to evaluate inflammation at the small joints of feet in a subset of psoriasis patients without clinical arthritis, using an office-based extremity MRI (eMRI) as compared to the findings in overt PsA patients.

Methods: Patients with psoriasis were recruited from Dermatology and Rheumatology clinics of a tertiary care institution in southern India were divided into those without arthritis ( $\mathrm{PsO}$ ) and PsA groups. All consenting patients underwent non-contrast eMRI of the right foot. Demographic and physical examination details were recorded. PsO patients completed the early arthritis in psoriasis (EARP) questionnaire. Two trained readers scored the MRI inflammation (synovitis, tenosynovitis, osteitis) using a modification of the PsAMRI scores (PsAMRIS). 1 Inter-reader agreement was assessed in a random subset of 42 cases using intra-class correlation coefficient (ICC). Proportion of patients with any sign of MRI inflammation was noted. Mann-Whitney $U$ test was used to compare inflammation scores of PsO with PsA patients. Clinical variables were compared with inflammation scores for any association.

Results: A total of 83 patients ( 30 PsA and $53 \mathrm{PsO}$ ) with $75 \%$ males and mean age of $42.2 \pm 11.6$ years were included. ICC for all three variables between the readers was very good $(>0.8)$. There was no statistical difference between the median eMRI inflammatory scores in PsA and PsO patients ( $\mathrm{p}=0.493$ ). Evidence of inflammation was present in $64 \%$ and $67 \%$ patients in the PsO and PsA groups, respectively (Table 1). Higher NAPSI scores were associated with presence of MRI inflammation $(\mathrm{p}=0.022)$.

Table 1. PsAMRIS variables for MRI inflammation of foot in PsO and PsA subgroups

\begin{tabular}{lcc}
\hline Variable & PsO $(\mathrm{n}=53)$ & $\mathrm{PsA}(\mathrm{n}=30)$ \\
\hline Synovitis & $34(64 \%)$ & $19(63 \%)$ \\
Osteitis & $2(4 \%)$ & $2(7 \%)$ \\
Flexor tenosynovitis & $9(17 \%)$ & $8(27 \%)$ \\
Inflammation & $34(64 \%)$ & $20(67 \%)$ \\
\hline
\end{tabular}

Conclusions: This study corroborates a high proportion of psoriasis patients with subclinical disease of the small joints of foot. Patients with nail involvement had a higher risk of subclinical disease. The cohort is being assessed longitudinally to determine the clinical utility of MRI feet in predicting subsequent development of PsA in patients with psoriasis.

References:

[1] Glinatsi D, Bird P, et al. Validation of the OMERACT psoriatic arthritis magnetic resonance imaging score (PSAMRIS) for the hand and foot in a randomized placebo-controlled trial. J Rheumatol 2015;42:2473-9.

Acknowledgements: This project is being funded by the APLAR and Christian Medical College, Vellore research board.

Disclosure of Interest: None declared

DOI: 10.1136/annrheumdis-2017-eular.5705

\section{FRI0630 CAN WHOLE BODY MRI AT BASELINE IDENTIFY DEFINITE INFLAMMATORY ARTHRITIS PATTERNS IN UNDIFFERENTIATED ARTHRITIS?}

J.E. Freeston ${ }^{1,2}$, K.S. Mankia ${ }^{1}$, M.-A. D'Agostino ${ }^{1,3}$, R. Hodgson ${ }^{2}$

A. Grainger ${ }^{2,4}$, I. Matsuura ${ }^{1}$, N. Larkman ${ }^{4}$, P.G. Conaghan ${ }^{1}$, P. Emery ${ }^{1}$

${ }^{1}$ LIRMM, University of Leeds; ${ }^{2}$ NIHR Leeds Musculoskeletal Biomedical

Research Centre, Leeds Teaching Hospitals NHS Trust, Leeds, United Kingdom;

${ }^{3}$ Université Versailles Saint Quentin en Yvelines, APHP Ambroise Paré,

Boulogne, France; ${ }^{4}$ Radiology, Leeds Teaching Hospitals NHS Trust, Leeds,

United Kingdom

Background: When diagnosing inflammatory arthritis (IA) early, focal joint imaging may not reflect the overall inflammatory burden/distribution. Whole body MRI (WBMRI) offers the potential to feasibly scan most joints in a single session. Objectives: The aims were (i) to describe the WBMRI pattern of disease in early IA (ii) to identify patterns associated with subsequent definite IA.

Methods: Patients were recruited with early inflammatory joint symptoms and/or signs of IA. Clinical data included age, gender, symptom duration, CRP, HLA-B27, RF, CCP Ab and tender/swollen joint counts. Using 3T WBMRI, T2-weighted fat suppressed spine/SIJ images pre contrast and 3D VIBE Dixon images of peripheral joints and entheses post IV contrast were acquired. Images were consensus scored for inflammation/erosion at the spine, SIJ, GHJ, SCJ, wrist, MCP, PIP, hip, knee, ankle, mid/hind foot, MTP and IP joints plus shoulder, ASIS, greater trochanter, knee, Achilles and plantar fascia entheses. Subjects were clinically classified at baseline and 1 year as undifferentiated arthritis (UA), CCP+RA, CCP-RA or Spondyloarthropathy $(\mathrm{SpA})$. Clinicians were unaware of the MRI findings.

Results: 39 patients (23 female) were recruited; mean age 43 years, median symptom duration 18 months $(7,24)$, TJC $5(2,11)$, SJC $1(0,3)$ and CRP $2(2,2)$. At baseline, 14 were classified as definite disease (RA or SpA) and 25 (14 female) as UA with mean age 40 years, median symptom duration 16.5 months $(9.8$, 24.3), TJC $3(1,8)$, SJC $0(0,1)$, CRP $2(2,2)$ and $3(12 \%)$ were HLA-B27 positive. The distribution of WBMRI findings in the classified (i.e. definite IA) group was predominantly small joint and tendon-based in the CCP+ RA group, large joint based with 50\% having SIJ disease in the CCP-group and similar findings in the SpA group. In the non-classified group (i.e. pUA and rUA), the distribution in pUA was both axial and peripheral, involving joints and entheses, with $25 \%$ having SIJ disease. In comparison, findings in the rUA group were similarly distributed but less frequent with no cases of SIJ disease. After 1 year of clinical/laboratory follow-up, 8 were identified as pUA, 6 rUA, 7 CCP+RA, 6 CCP-RA and 12 as $\mathrm{SpA}$. Table 1 shows WBMRI disease distribution by 1 year diagnostic category. The inclusion of affected WBMRI sites in the diagnostic work-up would have appropriately classified 6 further cases of definite SpA, 3 from the pUA and 3 from the CCP-RA groups.

Site of Disease Activity at Baseline on MRI

\begin{tabular}{lccccc}
\hline & \multicolumn{5}{c}{ Clinical Diagnostic category at 1 year } \\
\cline { 2 - 6 } & pUA $(\mathrm{n}=8)$ & rUA $(\mathrm{n}=6)$ & $\mathrm{CCP}+\mathrm{RA}(\mathrm{n}=7)$ & $\mathrm{CCP}-\mathrm{RA}$ & $\mathrm{SpA}(\mathrm{n}=12)$ \\
\hline Axial & $1(13 \%)$ & $1(17 \%)$ & $0(0 \%)$ & $1(17 \%)$ & $0(0 \%)$ \\
SIJ & $2(25 \%)$ & $0(0 \%)$ & $0(0 \%)$ & $3(50 \%)$ & $4(33 \%)$ \\
Large Joints & $3(38 \%)$ & $4(67 \%)$ & $4(57 \%)$ & $6(100 \%)$ & $6(50 \%)$ \\
Small Joints & $6(75 \%)$ & $2(33 \%)$ & $7(100 \%)$ & $5(83 \%)$ & $6(100 \%)$ \\
Tendons & $3(38 \%)$ & $1(17 \%)$ & $5(71 \%)$ & $5(83 \%)$ & $4(33 \%)$ \\
Entheses & $6(75 \%)$ & $3(50 \%)$ & $4(57 \%)$ & $5(83 \%)$ & $8(67 \%)$ \\
\hline
\end{tabular}

Key: pUA = Persistent UA, rUA $=$ Resolved UA, RA = Rheumatoid arthritis, SpA = Spondyloarthropathy. 
Conclusions: In the persistent UA group and CCP-RA group, WBMRI findings at baseline already showed a definite pattern of spinal disease. Therefore the use of WBMRI findings at presentation in addition to clinical assessment would allow clinicians to classify a proportion of patients earlier.

Disclosure of Interest: None declared

DOI: 10.1136/annrheumdis-2017-eular.5051

\section{FRI0631 PREDICTIVE VALUE OF POWER DOPPLER ULTRASONOGRAPHY (PDUS) IN THE DIAGNOSIS OF EARLY RHEUMATOID ARTHRITIS}

L. Mayordomo $^{1}$, C. Jurado ${ }^{2}$, M.L. Velloso ${ }^{1}$, A. Gutierrez-Leonard ${ }^{3}$,

P. González-Moreno ${ }^{4}$, J.L. Marenco ${ }^{1}$, C. Almeida ${ }^{5} \cdot{ }^{1}$ Rheumatology Department;

${ }^{2}$ Radiology Dp, Hospital Universitario Valme; ${ }^{3}$ Physioterapy, HVR;

${ }^{4}$ Rheumatology Department, HVM; ${ }^{5}$ Research and Statistics Dp, Hospital Universitario Valme, SEVILLA, Spain

Background: There is a short window of opportunity for early diagnosis and treatment of rheumatoid arthritis, that may be crucial for reaching remission and a low rate of radiographic progression. High resolution power doppler ultrasonography (PDUS) is helpful in early detection of synovitis and allows an accurate classification of patients with joint inflammation.

Objectives: To establish wether the presence of basal power doppler signal in patients with very early arthritis may be helpful in order to establish the risk of final diagnosis of rheumatoid arthritis according ACR criteria 1987 at a year of follow up.

Methods: We studied the presence of ultrasonographic Power Doppler (PD) signal on 28 joints (shoulders, elbows, wrists, MCPs, PIPs, knees) and 44 joints (28 joints and in addition hips, ankles, tarsus, and MTPs), with a mid-range equipment GE L5, in 70 patients with suspected early arthritis The patients were included with at least one of the following inclusion criteria: a) Swelling in 2 or more joints b) pain in MCPs, MTPs and/or the wrists c) morning stiffness of more than 30 minutes with $<12$ months of duration of the symptoms. Presence or not basal erosions (score $\geq 2$ in at least one joint by modified Sharp method) for each patient were registered (65 patients with basal hands and feet radiology available). Presence of RF and ACPA positive were recorded as well. At one year follow-up was established whether patients met criteria for RA according1987 ACR or not. Statistical study: Chi-square, Fisher exact test, p univariant and Odds Ratio calculation.

Results: The presence of basal power doppler signal in $\geq 1$ joints of 44 (PD44) in baseline visit shows statistically significant association to RA diagnosis at 12 months by ACR 1987 classification criteria, $p=0.003$, OR=5,43 $(1,71-17,24)$ but the presence of at least one joint with power doppler signal of 28 joints (PD28) did not $(\mathrm{p}=0.051)$. Presence hypertrophic synovium with PD44 or not, in at least one joint (HSORPD44) was associated to RA diagnosis as well $p=0.024$; OR $10,24(1,12-93,28)$. RF was positive in $18 / 70(25,71 \%)$ and was associated to RA diagnosis ( $\mathrm{p} 0.003$, OR $8,31(1,74-39,64)$, as well as FR/ACPA, positive in $20 / 70(28,57 \%), p=0.001$ OR $10(2,10-47,58)$. PD44 in addition to positive $\mathrm{RF}$ was associated to RA $p=0.003$, OR $12,93(1,59-104,94)$. Presence of basal radiographic erosions (BRE) was associated to $\mathrm{RA}, \mathrm{p}=0.001$ OR $7,72(2,2-26,8)$. PD44 in addition to BRE was significantly associated to RA $p=0.0005$, OR $29,33(3,61-238,37)$. PD28 in addition to RF or BRE was significantly associated to $R A$ respectively $p 0.0005$ OR $24,20(2,98-196.34)$ and $p=0.003$ OR 12,93 $(1,59-104,94)$.

\begin{tabular}{|c|c|c|c|c|c|}
\hline \multicolumn{2}{|l|}{ BASAL } & RA YES & $\stackrel{\text { P }}{ }$ & OR & Cl $95 \%$ \\
\hline PD 44 & $\begin{array}{l}\text { YES } \\
\text { NO }\end{array}$ & $\begin{array}{l}38 / 52(73,1 \%) \\
6 / 18(33,3 \%)\end{array}$ & 0.003 & 5,43 & $(1,71-17,24)$ \\
\hline PD44+FR & $\begin{array}{l}\text { YES } \\
\text { NO }\end{array}$ & $\begin{array}{l}15 / 16(93,9 \%) \\
29 / 54(53,7 \%)\end{array}$ & 0.003 & 12,93 & $(1,59-104,94)$ \\
\hline $\begin{array}{l}\text { PD44+BAS EROS } \\
n=65\end{array}$ & $\begin{array}{l}\text { YES } \\
\text { NO }\end{array}$ & $\begin{array}{l}24 / 25(96 \%) \\
18 / 40(45 \%)\end{array}$ & 0.0005 & 29,33 & $(3,61-238,37)$ \\
\hline HSORPD 44 & $\begin{array}{l}\text { YES } \\
\text { NO }\end{array}$ & $\begin{array}{c}43 / 64(67,19 \%) \\
1 / 6(16,6 \%)\end{array}$ & 0.024 & 10,24 & $(1,12-93,28)$ \\
\hline $\begin{array}{l}\text { BAS EROS } \\
n=65\end{array}$ & $\begin{array}{r}\text { YES } \\
\text { NO }\end{array}$ & $\begin{array}{l}26 / 30(86,7 \%) \\
16 / 35(45,7 \%)\end{array}$ & 0.001 & 7,72 & $(2,2-26,8)$ \\
\hline FR & $\begin{array}{l}\text { YES } \\
\text { NO }\end{array}$ & $\begin{array}{l}18 / 20(90 \%) \\
26 / 50(52 \%)\end{array}$ & 0.003 & 8,31 & $(1,74-39,64)$ \\
\hline FR/PCC & $\begin{array}{r}\text { YES } \\
\text { NO }\end{array}$ & $\begin{array}{c}20 / 22(90,9 \%) \\
24 / 48(50 \%)\end{array}$ & 0.001 & 10 & $(2,10-47,58)$ \\
\hline
\end{tabular}

Conclusions: The presence of at least one joint with power doppler signal of 44 joints (PD44) on baseline visit may help to predict the RA diagnosis at 12 months of follow up according to criteria ACR 1987, in patients with early RA. PD 44 in addition to classic RA factors (RF and basal radiographic erosions) increased the risk final of RA, till more than three times in case of concomitant basal PD44 and radiographic erosions.

Disclosure of Interest: None declared

DOI: 10.1136/annrheumdis-2017-eular.6282

\section{FRI0632 MRI-US FUSION IMAGES FOR RHEUMATOID ARTHRITIS: CAN DOPPLER SUBSTITUTE FOR GADOLINIUM?}

F. Barbieri ${ }^{1}$, A. Muda ${ }^{2}$, S. Migone ${ }^{2}$, V. Prono ${ }^{2}$, I. Mussetto ${ }^{2}$, N. Romano ${ }^{2}$, A. Fischetti ${ }^{3}$, V. Tomatis ${ }^{1}$, S. Paolino ${ }^{4}$, M. Cutolo $^{4}$, G. Garlaschi ${ }^{2}$,

M.A. Cimmino ${ }^{1} .{ }^{1}$ Dipartimento di Medicina Interna, Clinica Reumatologica;

${ }^{2}$ Sezione di Radiologia; ${ }^{3}$ Università di Genova; ${ }^{4}$ Clinica Reumatologica, Genova, Italy

Background: $\mathrm{MRI}$ is increasingly used to objectively assess disease activity and damage in patients with rheumatoid arthritis (RA), especially in clinical trials. The preferred scoring method is the RAMRIS, which implies the use of gadolinium, an intravenous contrast agent, to assess synovitis.

Objectives: We evaluated in a small preliminary study if the fusion of MRI and US Power Doppler (PD) images could avoid gadolinium.

Methods: 12 patients (10 women) affected by RA, with at least one involved wrist, were studied. Mean age was $58.8 \pm 9.1$ years and mean disease duration was $54.5 \pm 56.1$ months. Disease activity (DAS 28-CRP) was evaluated on the day of the examinations.

MRI was performed on a extremity-dedicated machine (Oscan $0.3 \mathrm{~T}$, Esaote, Genova Italy) and US by MyLab Twice ultrasound scanner with a virtual navigator software (Esaote, Genova, Italy). T3Dt1 sequences were used in the fusion images (parameters: TR/TE/NEX 38/16/1, matrix 192*192*39, FOV 160*160*60, thickness $0.6 \mathrm{~mm}$ ). US scans were performed sagittally on the radio-lunate joint and axially on the proximal carpal bones of the dorsal wrist with B-mode and PD (PRF $500-750 \mathrm{~Hz}$; WF: 3; maximum gain with probe's calibration in air). Magnetic position sensor linked to the US transducer $(13 \mathrm{MHz})$ and low magnetic field transmitters were used to allowed fusion images.

MRI synovitis was scored by the RAMRIS and with the contrast-enhanced dynamic method resulting in rate of early enhancement (REE) and relative enhancement, (RE). The US score was the Global OMERACT-EULAR Synovitis Score (GLOESS) and its individual parts.

Results: Inter-reader agreement for PD by weighted kappa was $0.75(75 \% \mathrm{Cl}$ $0.53-0.96)$ for the sagittal and $1(75 \% \mathrm{Cl} 1-1)$ for the axial view. It was 0.85 (75\% Cl 0.69-0.98) for MRI synovitis.

There was a correlation between DAS28 and the MRI synovits score $(r=0.59$, $p=0.04)$, REE $(r=0.60, p=0.04)$, and $R E(r=0.58, p=0.05)$, US Doppler in the long $(r=0.75, p=0.005)$ and in the axial axes $(r=0.86, p=0.01)$, but not with B-mode synovial effusion $(r=0.56, p=0.056)$. The GLOESS correlated with DAS28 $(r=0.66$, $\mathrm{p}=0.019$ ), but not with RAMRIS synovitis, erosions or BME nor with REE. The MRI wrist synovitis score did not correlate with the Doppler score on the sagittal and axial views, nor with US B mode score. This was true also for the three individual MRI slices of the wrist, the REE and the RE. The figure shows from the left the pre-contrast axial MRI image, the post-contrast and the fusion images.

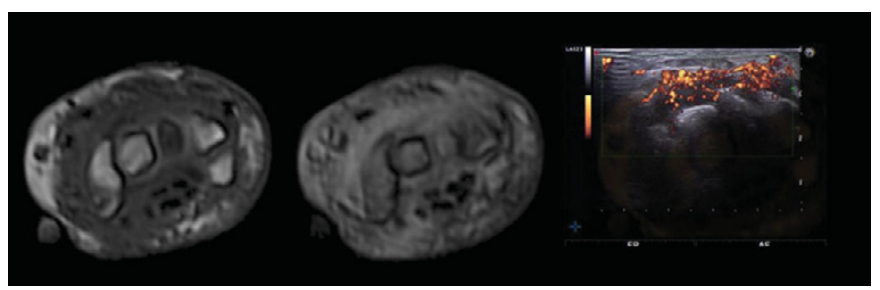

Conclusions: Although both MRI and Doppler are good indicators of disease activity in RA, they seem not to be interchangeable. This may be due to the fact that MRI and US show different features of synovitis or, alternatively, that MRI comprised the whole wrist whereas US visualized only its dorsal area. Although our preliminary data do not support the use of fusion images, these should be investigated on larger number of patients with a more extended PD approach.

Disclosure of Interest: None declared

DOI: 10.1136/annrheumdis-2017-eular.5433

\section{FRI0633 WHICH ARE THE ULTRASOUND LESIONS UNDERLYING DACTYLITIS?}

A. Nzeusseu Toukap ${ }^{1,2}$, T. Kirchgesner ${ }^{3}$, F. Lecouvet ${ }^{1,3}$, P. Navarro ${ }^{2}$, B. Vande Berg $^{1,3}$, A. Durnez ${ }^{2}$, M.S. Stoenoiu ${ }^{1,2}{ }^{1}$ Institut de Recherche Expérimentale et Clinique (IREC), Université catholique de Louvain; ${ }^{2}$ Rheumatology department; ${ }^{3}$ Radiology department, Cliniques Universitaires Saint-Luc, Brussels, Belgium

Background: Dactylitis, defined as a diffuse swelling of a digit is a hallmark feature of peripheral spondyloarthritis $(\mathrm{SpA})$, particularly in psoriatic arthritis, with a prevalence between $16 \%$ and $48^{1}$

Objectives: This study aims to assess the frequency of the pathological lesions in dactylitis using ultrasonography (US) and to evaluate their association with patient-reported tenderness.

Methods: Thirty-four dactylitis from 20 consecutive patients suffering from peripheral spondyloarthritis were examined by ultrasound. At US examination, the entire digit was scanned both on dorsal and palmar/plantar sides. The following US pathological lesions were scored: soft tissue thickness, soft tissue edema, soft tissue vascularization, synovitis of metacarpophalangeal (MCP)/metatarsophalangeal (MTP), of proximal interphalangeal (PIP) and of 